\title{
Exercise-induced BCL2-regulated autophagy is required for muscle glucose homeostasis
}

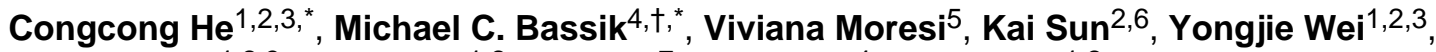 \\ Zhongju Zou ${ }^{1,2,3}$, Zhenyi An ${ }^{1,2}$, Joy Loh ${ }^{7}$, Jill Fisher ${ }^{4}$, Qihua Sun ${ }^{1,2}$, Stanley Korsmeyer ${ }^{4, \ddagger}$, \\ Milton Packer ${ }^{8}$, Herman I. May ${ }^{2}$, Joseph A. Hill ${ }^{2}$, Herbert W. Virgin ${ }^{7}$, Christopher Gilpin ${ }^{9}$, \\ Guanghua $\mathrm{Xiao}^{8}$, Rhonda Bassel-Duby ${ }^{5}$, Philipp E. Scherer ${ }^{2,6}$, and Beth Levine ${ }^{1,2,3,10}$ \\ ${ }^{1}$ Center for Autophagy Research, University of Texas Southwestern Medical Center, 5323 Harry \\ Hines Boulevard, Dallas, Texas 75390, USA \\ ${ }^{2}$ Department of Internal Medicine, University of Texas Southwestern Medical Center, 5323 Harry \\ Hines Boulevard, Dallas, Texas 75390, USA \\ ${ }^{3}$ Howard Hughes Medical Institute, University of Texas Southwestern Medical Center, 5323 Harry \\ Hines Boulevard, Dallas, Texas 75390, USA \\ ${ }^{4}$ Dana-Farber Cancer Institute, Massachusetts 02115, USA \\ ${ }^{5}$ Department of Molecular Biology, University of Texas Southwestern Medical Center, 5323 Harry \\ Hines Boulevard, Dallas, Texas 75390, USA \\ ${ }^{6}$ Touchstone Diabetes Center, University of Texas Southwestern Medical Center, 5323 Harry \\ Hines Boulevard, Dallas, Texas 75390, USA \\ ${ }^{7}$ Department of Pathology and Immunology, Washington University School of Medicine, St Louis, \\ Missouri 6110, USA \\ ${ }^{8}$ Department of Clinical Sciences, University of Texas Southwestern Medical Center, 5323 Harry \\ Hines Boulevard, Dallas, Texas 75390, USA \\ ${ }^{9}$ Department of Cell Biology, University of Texas Southwestern Medical Center, 5323 Harry Hines \\ Boulevard, Dallas, Texas 75390, USA \\ ${ }^{10}$ Department of Microbiology, University of Texas Southwestern Medical Center, 5323 Harry \\ Hines Boulevard, Dallas, Texas 75390, USA
}

\section{Abstract}

Exercise has beneficial effects on human health, including protection against metabolic disorders such as diabetes ${ }^{1}$. However, the cellular mechanisms underlying these effects are incompletely understood. The lysosomal degradation pathway, autophagy, is an intracellular recycling system that functions during basal conditions in organelle and protein quality control ${ }^{2}$. During stress, increased levels of autophagy permit cells to adapt to changing nutritional and energy demands

\footnotetext{
Correspondence and requests for materials should be addressed to B.L. (beth.levine@utsouthwestern.edu)..

*These authors contributed equally to this work.

†Present address: University of California at San Francisco, San Francisco, California 94158, USA.

\$Deceased.

Supplementary Information is linked to the online version of the paper at www.nature.com/nature.

Author Contributions C.H., M.C.B., V.M., K.S., S.K., M.P., J.A.H., H.W.V., R.B.-D., P.E.S. and B.L. designed the experiments. C.H., M.C.B., V.M., K.S., Y.W., Z.Z., Z.A., J.L., J.F., Q.S., H.I.M. and C.G. performed the experiments. G.X. performed statistical analyses. C.H. and B.L. wrote the manuscript.

The authors declare no competing financial interests.
} 
through protein catabolism ${ }^{3}$. Moreover, in animal models, autophagy protects against diseases such as cancer, neuro-degenerative disorders, infections, inflammatory diseases, ageing and insulin resistance ${ }^{4-6}$. Here we show that acute exercise induces autophagy in skeletal and cardiac muscle of fed mice. To investigate the role of exercise-mediated autophagy in vivo, we generated mutant mice that show normal levels of basal autophagy but are deficient in stimulus (exercise- or starvation)-induced autophagy. These mice (termed BCL2 AAA mice) contain knock-in mutations in BCL2 phosphorylation sites (Thr69Ala, Ser70Ala and Ser84Ala) that prevent stimulus-induced disruption of the BCL2-beclin-1 complex and autophagy activation. BCL2 AAA mice show decreased endurance and altered glucose metabolism during acute exercise, as well as impaired chronic exercise-mediated protection against high-fat-diet-induced glucose intolerance. Thus, exercise induces autophagy, BCL2 is a crucial regulator of exercise- (and starvation)- induced autophagy in vivo, and autophagy induction may contribute to the beneficial metabolic effects of exercise.

To investigate whether autophagy is induced by exercise, we analysed tissues of mice that transgenically express a green fluorescent protein (GFP)-labelled marker of autophagosomes, GFP-LC3 (LC3 is also known as MAP1LC3) (ref. 7), after treadmill exercise. In both skeletal and cardiac muscle, autophagosome (GFP-LC3 puncta) numbers increased after $30 \mathrm{~min}(\sim 300 \mathrm{~m})$ of running and reached a plateau at $80 \mathrm{~min}(\sim 900 \mathrm{~m})$ (Fig. $1 \mathrm{a}-\mathrm{c})$. This was observed in several muscle groups, including the vastus lateralis (thigh muscle) (Fig. 1a-c), soleus, tibialis anterior and extensor digitorum longus (Supplementary Fig. 1). Exercise also resulted in biochemical evidence of skeletal and cardiac muscle autophagy, including conversion of the non-lipidated form of LC3, LC3-I, to the autophagosome-membrane-associated lipidated form, LC3-II, and degradation of the autophagy substrate protein p62 (Fig. 1d). In addition, exercise induced autophagy in other organs involved in glucose and energy homeostasis, such as liver and pancreas, including islet $\beta$-cells (Supplementary Fig. 2a-af) and (based on LC3-II conversion) adipose tissue (Supplementary Fig. 2g). Thus, exercise is a newly defined stimulus that induces autophagy in vivo.

We found that exercise-induced autophagy in vivo involves disruption of the BCL2-beclin-1 complex. BCL2 is an anti-apoptotic and anti-autophagy protein that inhibits autophagy through a direct interaction with the $\mathrm{BH} 3$ domain of the autophagy protein beclin 1 at the endoplasmic reticulum ${ }^{8}$. Disruption of the BCL2-beclin-1 complex is crucial for stimulusinduced autophagy in mammalian cells ${ }^{8}$. Beclin 1 immunoprecipitation with BCL2 decreased in muscle within 15 min of exercise and was barely detectable after $30 \mathrm{~min}$ (Fig. 1e). Dissociation of the BCL2-beclin-1 complex was not associated with phosphorylation of kinases previously linked to BCL2 phosphorylation, such as JNK and p38 MAPK ${ }^{9}$ (Supplementary Fig. 3).

To study the physiological functions of exercise-induced autophagy in vivo, we constructed mutant mice that are deficient in stimulus-induced but not basal autophagy. As phosphorylation of three sites in the non-structured loop of human BCL2 is critical for stimulus-induced autophagy in vitro ${ }^{8}$, we generated knock-in $\left(B c l 2^{\mathrm{AAA}}\right)$ mice lacking three conserved phosphorylation residues in the non-structured loop region of mouse BCL2: Thr 69, Ser 70 and Ser 84 (homologous to human Ser 87) were replaced by alanines (BCL2 AAA) (Supplementary Fig. $4 \mathrm{a}-\mathrm{c}$ ). Homozygous $\mathrm{Bcl} 2^{\mathrm{AAA}}$ mice were viable and fertile, born in the expected Mendelian ratio, of normal size and weight, and displayed normal histology of major organs (data not shown). Steady-state levels of BCL2 AAA in Bcl2 $2^{\mathrm{AAA}}$ mice were similar to those of wild-type (WT) BCL2 in Bcl2 ${ }^{\mathrm{WT}}$ mice in skeletal and cardiac muscle, liver, adipose tissue and pancreas (Supplementary Fig. 4d). 
Murine embryonic fibroblasts (MEFs) derived from BCL2 AAA mice showed a defect in stimulus-induced autophagy (Fig. 2a, b). In response to starvation, BCL2 underwent phosphorylation, BCL2 dissociated from beclin 1, and autophagy was induced in MEFs from isogenic wild-type mice. However, in BCL2 AAA MEFs, BCL2 phosphorylation was absent (as measured by p32 labelling), the BCL2-beclin-1 complex was not disrupted, and less autophagy was induced. Levels of basal autophagy were similar in wild-type and BCL2 AAA MEFs. Similar findings were observed in vivo in skeletal and cardiac muscle (Supplementary Fig. 5); autophagosomenumbers were similar at baseline in wild-type and BCL2 AAA mice expressing GFP-LC3 but failed to increase in GFP-LC3 BCL2 AAA mice in response to $48 \mathrm{~h}$ starvation.

To evaluate whether BCL2 AAA mice are deficient in exercise-induced autophagy, we exercised GFP-LC3 wild-type mice and GFP-LC3 BCL2 AAA mice for a fixed time and fixed distance ( $80 \mathrm{~min}(\sim 900 \mathrm{~m})$ ) and at $75 \%$ of their maximal running capacity (see Fig. $3 a)$. Under both conditions, BCL2 AAA mice displayed marked impairment of exerciseinduced upregulation of skeletal and cardiac muscle (as measured by numbers of GFP-LC3 puncta) (Fig. 2c, d and Supplementary Fig. 1b). BCL2 AAA mice also exhibited less exercise-induced LC3-II conversion and p62 degradation in skeletal and cardiac muscle (Fig. 2e), impaired autophagic responses in liver and pancreatic $\beta$-cells (Supplementary Fig. 2c, f), and defectiveexercise-induced dissociation of the BCL2-beclin-1 complex in muscle (Fig. 2f). Thus, non-phosphorylatable BCL2 does not alter basal autophagy in vivo, but prevents autophagy activation in response to starvation and exercise. This blockade of autophagy activation was not associated with increased cell death (Supplementary Fig. 6).

The deficient exercise-induced autophagy in BCL2 AAA mice was accompanied by lower maximal exercise capacity (Fig. 3a). BCL2 AAA mice had similar levels of serum lactate after maximal exercise, similar baseline muscle strength, and similar baseline cardiac function and size as wild-type mice (Supplementary Fig. 7). Characterization of various muscle groups (soleus, tibialis anterior and extensor digitorum longus) from wild-type and BCL2 AAA mice at baseline and after $80 \mathrm{~min}$ of exercise revealed no differences in weight, fibre cross-sectional area, morphology (haematoxylin and eosin staining), fibre type analysis, glycogen content, or mitochondrial content and functionality (Fig. $3 \mathrm{~b}$ and Supplementary Figs 8-10). These analyses suggest that differences in baseline cardiac and skeletal muscle properties do not account for decreased exercise endurance in BCL2 AAA mice.

During strenuous exercise, mammals undergo metabolic changes to increase skeletal muscle glucose uptake/utilization efficiency, including increased insulin sensitivity and redistribution of glucose transporters, such as GLUT4 (also known as SLC2A4), to the plasma membrane ${ }^{10,11}$, which is essential for exercise-stimulated glucose uptake ${ }^{12}$. However, BCL2 AAA mice demonstrated impaired exercise-induced increase in insulin sensitivity, as reflected by less of a decline in plasma glucose (Fig. 3c) and plasma insulin (Fig. 3d) levels than in wild-type mice. Also, unlike wild-type mice, BCL2 AAA mice failed to exhibit increased plasma membrane GLUT4 localization in the vastus lateralis and soleus muscles following maximal or 80 min exercise, respectively (Fig. 3e and Supplementary Figs 11a and 12a) and they exhibited lower levels of radiolabelled glucose uptake in soleus muscle (Fig. 3h).

Adenosine monophosphate-activated protein kinase (AMPK) has a central role in enhanced GLUT4 plasma membrane localization and skeletal muscle glucose uptake during exercise $^{13}$. BCL2 AAA mice had a notable decrease in AMPK activation, as measured by levels of phosphorylation at residue threonine 172, in both the vastus lateralis (Fig. $3 \mathrm{f}$ and Supplementary Fig. 11b) and in the soleus (Supplementary Fig. 12b) muscles. 
Phosphorylation of the downstream AMPK target acetyl-CoA carboxylase (ACC) paralleled AMPK phosphorylation (Supplementary Fig. 13), providing additional support for a defect in exercise-induced AMPK activation in BCL2 AAA mice.

No differences were observed in phosphorylation of CaMK1, a downstream target of the $\mathrm{Ca}^{2+}$-stimulated kinase CaMKK $\beta$ (which is upstream of AMPK in certain contexts) ${ }^{13}$ (Supplementary Fig. 13b), suggesting that differences in levels of AMPK activation between wild-type and BCL2 AAA mice are not due to altered $\mathrm{Ca}^{2+}$ homeostasis. They are also probably unrelated to possible effects of BCL2 on mitochondrial function, because phosphorylated BCL2 resides at the endoplasmic reticulum ${ }^{14}$ and markers of mitochondrial function did not differ in the muscles of BCL2 AAA and wild-type mice (Supplementary Fig. 10). Differences in insulin signalling molecules also did not explain the altered glucose homeostasis in exercised BCL2 AAA mice, because at late time points during exercise, decreased (not increased) AKT activation and mTOR activation was observed in muscle of both wild-type and BCL2 AAA mice (Supplementary Fig. 13a). No differences were observed in muscle phosphorylation of the AKT substrate AS160 (also known as TBC1D4), or levels of the muscle-specific forkhead transcription factor target atrogin 1 (also known as FBXO32), in response to exercise or between genotypes.

To confirm that the observed defects in exercise-induced endurance and muscle glucose metabolism are due to defective autophagy, we examined mice with allelic loss of the autophagy gene beclin 1 (BecnI), which have decreased beclin 1 protein expression and decreased starvation-induced autophagy in skeletal muscle ${ }^{15}$. Similar to BCL2 AAA mice, $B e c n 1^{+/-}$mice exhibited deficient exercise-induced autophagy in skeletal muscle (Supplementary Fig. 14a, b), normal muscle strength (Supplementary Fig. 14c), decreased maximal treadmill running distance (Fig. 3g), impaired exercise-induced GLUT4 plasma membrane localization (Supplementary Fig. 14d) and muscle glucose uptake (Fig. 3h), and lower levels of exercise-induced muscle AMPK activation (phospho-AMPK and its target, phospho-ACC) (Supplementary Fig. 14e) than wild-type controls. The similarity of the phenotypes of BCL2 AAA and Becn ${ }^{+/-}$mice provides strong support for a role of deficient beclin 1 activity, rather than other BCL2-regulated functions, in the impairment of exercise endurance, glucose uptake and AMPK activation in BCL2 AAA mice.

AMPK activates autophagy in response to low energy conditions by sensing the cellular ratio of AMP to ATP ${ }^{13}$. Deficient AMPK activation in BCL2 AAA and Becn ${ }^{+/-}$mice during exercise raised the possibility that, similar to observations in vitro ${ }^{16}$, the autophagy pathway may function in vivo in a feed-forward manner in AMPK activation. To evaluate whether impaired exercise-induced AMPK activation in vivo is due to deficient autophagy, rather than potential autophagy-independent effects of the BCL2 AAA mutation or monoallelic loss of beclin 1, we examined exercise-induced AMPK activation in $\operatorname{Atg}_{161}{ }^{\mathrm{HM}}$ mice that are hypomorphic for ATG16L1 (ref. 17), an essential autophagy protein that specifies the LC3 lipidation site for autophagosomal membrane biogenesis ${ }^{18} \cdot \operatorname{Atg}_{1611^{\mathrm{HM}}}$ mice had a significant reduction in ATG16L1 protein expression in skeletal muscle and a defect in exercise-induced autophagy in muscle lysates after $80 \mathrm{~min}$ of exercise (Supplementary Fig. 15a, b). This defect in exercise-induced autophagy was associated with decreased exercise-induced AMPK phosphorylation and ACC phosphorylation (Supplementary Fig. 15c).

Together, our in vivo studies in BCL2 AAA, Becn $1^{+/-}$and $A \operatorname{tg} 1611^{\mathrm{HM}}$ mice suggest that cellular autophagy function is partially required for normal levels of exercise-induced muscle AMPK activation. The mechanism underlying this requirement is not yet known, but is unlikely to be caused by non-specific effects of the mouse mutations on AMPK activation as the direct AMPK activator, the AMP analogue 5-aminoimidazole-4-carboxamide riboside 
(AICAR), induced AMPK activation normally in BCL2 AAA, Becn $1^{+/-}$and $\operatorname{Atg} 1611^{\mathrm{HM}}$ MEFs (Supplementary Fig. 16).

We next examined whether exercise-induced autophagy is required for thebeneficialmetabolic effectsoflong-term exercisetraining, using a high-fat diet (HFD) model of obesity and impaired glucose tolerance ${ }^{6,19}$. Age-matched cohorts of wild-type andBCL2 AAA mice werefed a HFD for four weeks; the HFD was continued for an additional 8 weeks either combined with 50 min of daily treadmill exercise or with no exercise (Supplementary Fig. 17a). In both wild-type and BCL2 AAA mice, HFD led to body weight gain (Supplementary Fig. 17b), which was probably due to increased fat mass (Supplementary Fig. 18a), as no differences in lean mass or muscle fibre size were observed (Supplementary Fig. 18). HFD led to modest suppression of baseline autophagy in skeletal and cardiac muscle as measured by levels of LC3-II conversion and p62 in tissue lysates (Supplementary Fig. 19). Ultrastructural analyses of the liver and pancreas did not reveal any differences in HFD versus regular diet animals, in wild-type versus BCL2 AAA mice, or in non-exercised versus exercised animals, except for hepatic lipid droplet accumulation in all HFD-fed groups (Supplementary Fig. 20). Although BCL2 AAA mice were more sensitive to HFD-induced obesity, 8 weeks of daily exercise reduced the magnitude of weight gain similarly in BCL2 AAA and wild-type mice (Supplementary Fig. 17b). Thus, the BCL2 AAA mutation did not alter the response of mice to HFD with respect to muscle fibre size, the morphology of liver and pancreas, or the effect of exercise on HFD-induced obesity.

However, BCL2 AAA mice failed to exhibit normal exercise-induced protection against HFD-induced impaired glucose tolerance. Before the administration of HFD, wild-type and BCL2 AAA mice had similar clearance rates in oral glucose tolerance tests (Fig. 4a). Four weeks of HFD led to impaired glucose tolerance in both genotypes versus regular diet control groups (Fig. 4b); the magnitude of this impairment was similar in BCL2 AAA and wild-type mice despite more weight gain in the BCL2 AAA mice. However, exercise training markedly improved glucose tolerance in HFD-fed wild-type mice but not in autophagy-deficient BCL2 AAA mice (Fig. 4c and Supplementary Fig. 21). This failure of BCL2 AAA mice to show improved glucose tolerance is unlikely to be due to deficient insulin production, because at the end of the study HFD-fed wild-type and BCL2 AAA mice displayed similar circulating fasting insulin levels (Supplementary Fig. 22a), similar levels of insulin secretion in response to oral glucose challenge (Supplementary Fig. 22b), and similar pancreatic $\beta$-cell morphology (Supplementary Fig. 20b). These results suggest that BCL2-regulated functions are essential for chronic exercise-mediated protection against HFD-induced glucose intolerance. We speculate that, as in acute exercise, this may involve the dynamic interplay of autophagy induction and AMPK activation.

HFD-induced obesity is associated with several other metabolic changes, including increased levels of serum leptin, an appetite-inhibiting adipokine $;{ }^{20}$ reduced levels of circulating adiponectin ${ }^{21}$, an antidiabetic adipokine ${ }^{22-24}$; and increased serum triglycerides and cholesterol. In HFD-fed mice, baseline levels of leptin were higher in BCL2 AAA mice than in wild-type mice, and 8 weeks of exercise decreased serum leptin in wild-type but not in BCL2 AAA mice (Fig. 4d). Although adiponectin levels in HFD-fed mice were similar in the non-exercised wild-type and BCL2 AAA groups, they were significantly increased in the exercised wild-type mice versus the exercised BCL2 AAA mice (Fig. 4d). Both serum triglycerides and cholesterol increased in wild-type and BCL2 AAA HFD-fed mice; with exercise, the values returned to those observed in animals fed a regular diet in wild-type but not BCL2 AAA mice (Supplementary Fig. 23). Thus, the BCL2 AAA mutation impaired the beneficial effects of exercise on metabolic changes induced by a HFD. 
The HFD-fed, daily exercised wild-type mice weremore metabolically active than their BCL2 AAA counterparts, as demonstrated by elevated levels of oxygen consumption, $\mathrm{CO}_{2}$ production and heat generation during a $12 \mathrm{~h}$ night interval when the mice were not exercised (Supplementary Fig. 24a). In addition, in vastus lateralis muscle, wild-type mice had elevated induction of messenger RNA for UCP1, Ucp1, a mitochondrial uncoupling protein mainly expressed in brown adipose tissue that contributes to thermogenesis and energy expenditure ${ }^{25}$ (Supplementary Fig. 24d). These changes were probably due to improved metabolic fitness in response to exercise rather than alterations in food intake, spontaneous physical activity, or general mitochondrial function. No differences were observed in daily food intake in exercised HFD-fed wild-type and BCL2 AAA mice (Supplementary Fig. 24b). Spontaneous physical activity was similar in HFD-fed nonexercised wild-type and BCL2 AAA mice, although there was a trend towards decreased spontaneous physical activity in the exercised BCL2 AAA mice (Supplementary Fig. 24c). No changes were observed with exercise in either genotype in mRNA levels of the skeletal muscle-expressed uncoupling proteins UCP2 or UCP3, or the mitochondrial proteins cytochrome $b$ and $c$ (Supplementary Fig. 24d, e).

The HFD study suggests that increased autophagy triggered by exercise may be critical for improving impaired glucose tolerance and metabolism in diet-induced obesity. However, we cannot definitely conclude that lack of exercise-induced improvement in glucose tolerance in HFD-fed BCL2 AAA mice is caused by deficient exercise-induced autophagy; it is possible that other effects of the BCL2 AAA mutation are responsible for this phenotype. Nonetheless, given our findings in acute exercise (which demonstrate impaired muscle glucose uptake, GLUT4 plasma membrane localization and AMPK activation in autophagydeficient animals), it seems plausible that alterations in exercise-induced skeletal muscle glucose metabolism in autophagy-deficient animals may also contribute to the failure of exercise to reverse HFD-induced metabolic abnormalities.

Our findings demonstrate that exercise is a potent inducer of autophagy, and that acute and chronic exercise enhances glucose metabolism in mice capable of inducing autophagy but not in autophagy-deficient mice. These beneficial metabolic effects (as well as exercise- and starvation-induced autophagy) are blocked by a mutation in BCL2 that prevents its release from an inhibitory interaction with the autophagy protein beclin 1. Thus, BCL2 has previously undescribed essential roles in the in vivo regulation of stimulus-induced autophagy as well as glucose metabolism. We propose that BCL2-regulated autophagy activation contributes to the beneficial metabolic effects of exercise, and that manipulation of the autophagy pathway and/or the function of the autophagy inhibitory BCL2 protein may be a logical strategy to mimic the health effects of exercise and to prevent or treat impaired glucose metabolism. More broadly, on the basis of this newly discovered link between exercise, autophagy and altered metabolism, we speculate that autophagy may represent a cellular mechanism by which exercise prolongs life and protects against cancer, cardiovascular disorders and inflammatory diseases ${ }^{1}$.

\section{METHODS SUMMARY}

\section{Mouse strains}

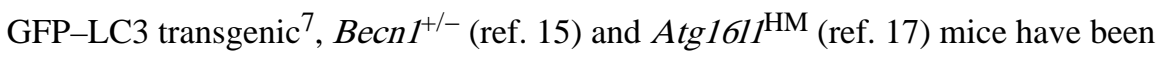
described. The generation of mice with a knock-in mutation in $B c 12$ that results in Thr69Ala, Ser70Ala and Ser84Ala mutations in BCL2 (BCL2 AAA) is described in Methods. 


\section{Exercise studies}

Acute and chronic exercise studies were performed using a treadmill protocol described in Methods.

\section{Autophagy assays}

Autophagy was measured by visualizing GFP-LC3 puncta by fluorescence microscopy in MEFs or tissue sections from mice with transgenic expression of GFP-LC3, or by western blot analysis of LC3-II conversion and p62 levels in tissue lysates.

\section{Metabolic analyses}

Metabolic and blood parameters in the acute and chronic exercise studies were measured as described in Methods.

\section{METHODS}

\section{Mouse strains}

GFP-LC3 transgenic, Becn $1^{+/-}$and $A \operatorname{tg} 1611^{\mathrm{HM}}$ mice have been previously described ${ }^{7,15,17}$. For the construction of a mouse strain with knock-in mutations in the phosphorylation sites in the non-structured loop of BCL2 ( $B c 12^{\mathrm{AAA}}$ mice), BAC clones (Incyte) were screened for the presence of $B c l 2$ using the following primers: BCL2 $5^{\prime}$,

GTGGGGCGGGAGTCGGGACT; BCL2 3', GACCCAGAATCCACTCACAC. The Bcl2

BAC clone was digested by BglII, subcloned into pSP72, and a puromycin resistance marker flanked by FRT sites (SalI fragment of pPGKPuro, cloned into pFRT) was blunt cloned into a BsaBI site $3^{\prime}$ of exon II. A mutant allele was generated by subcloning a PstI fragment from the coding region. Amino acid residues Thr 69, Ser 70 and Ser 84 (homologous to human Ser 87) were changed to alanine in two steps using the Quickchange PCR (Stratagene) and the following primer sets: TSAA 5', GAGATGGCTGCCA GGGCGGCTCCTCTCAGGCCCC; TSAA 3', GGGGCCTGAGAGGAGCC GCCCTGGCAGCCATCTC; S84A 5', GCTGGGCCTGCGCTCGCCCC TGTGC CACCATG; S84A 3', CATGGTGGCACAGGGGCGAGCGCAGGCCCAGC. The targeting construct 7.1AAA was electroporated into RW4 embryonic stem cells (129 Sv/J), and $36 \mathrm{~h}$ later, clones were selected with puromycin, picked and screened by Southern blot analysis with the probes indicated in Supplementary Fig. $4 \mathrm{a}$. A $1.7 \mathrm{~kb}$ shift upwards indicative of the mutant allele was detected on Southern blots in 2/300 clones as the targeted locus (Supplementary Fig. 4b). These clones were tested for normal karyotype and used to inject blastocysts from C57BL/6J donors. Mice with germline transmission were bred to mice expressing FLP from the $\beta$-actin promoter (Jackson Laboratories: B6;

SJLTgN(ACTFLPe)9205Dym), and offspring were screened for deletion of the puromycin selection marker. To screen for the presence of the mutation following excision of the selectable marker, $\mathrm{PCR}$ with $5^{\prime}$ and $3^{\prime} B c l 2$ primers was performed, and digestions were performed with BgII or AatII enzymes; BglI cuts the amplified fragment only if the T69AS70A site is present, while AatII cuts only the wild-type sequence for the same region (Supplementary Fig. 4c). Using this scheme, homozygous $B c 12^{\mathrm{AAA}}$ mutants were identified by the presence of PCR fragments cleaved only by BgII and not AatII, whereas wild-type mice were identified by the presence of PCR fragments cleaved only by AatII and not BglI. $B c 12^{\mathrm{AAA}}$ mice were backcrossed for more than ten generations to C57/B6 mice (Jackson Laboratories), and homozygous $B c l 2^{\mathrm{AAA}}$ and $B c l 2^{\mathrm{WT}}$ offspring were used in all studies. 


\section{Cell lines}

Primary MEFs were established either from $B c 2^{\mathrm{AAA}}$ and $B c 2^{\mathrm{WT}}$ mice, the offspring of $B c l 2^{\mathrm{AAA}}$ and $B c 2^{\mathrm{WT}}$ mice crossed with GFP-LC3 transgenic mice, $B e c n 1^{+/-}$and $B e c n 1^{+/+}$ mice, and $A \operatorname{tg} 1611^{\mathrm{HM}}$ and $A \operatorname{tg} 1611^{\mathrm{WT}}$ at embryonic day 13.5 and cultured as described ${ }^{26}$.

\section{Radiolabelling and co-immunoprecipitation from MEFs}

MEFs derived from $B c / 2^{\mathrm{AAA}}$ and $\mathrm{Bcl} 2^{\mathrm{WT}}$ mice were cultured overnight in labelling medium (phosphate-free DMEM with 10\% dialysed FBS) and then cultured in starvation medium (EBSS minus phosphate) or labelling medium for $4 \mathrm{~h}$ containing $2 \mathrm{mCi} \mathrm{ml}^{-1}{ }^{32} \mathrm{P}-$ orthophosphate. Cells were lysed in lysis buffer containing $50 \mathrm{mM}$ Tris (pH 7.9), $150 \mathrm{mM}$ $\mathrm{NaCl}, 1 \mathrm{mM}$ EDTA, $1 \%$ Triton X-100, proteinase inhibitor cocktail (Roche Applied Sciences) and halt phosphatase inhibitor cocktail (Thermo Scientific), and were subjected to immunoprecipitation with a monoclonal anti-BCL2 antibody (Santa Cruz Biotechnology, 1:50). Eluates were separated by SDS-PAGE and detected by anti-beclin 1 antibody (Santa Cruz Biotechnology, 1:200), anti-BCL2-HRP antibody (C2, Santa Cruz Biotechnology, $1: 100)$ and autoradiography.

\section{Co-immunoprecipitations from muscle tissue}

Vastus lateralis (thigh muscle) was dissected and homogenized in lysis buffer containing 50 $\mathrm{mM}$ Tris (pH 7.9), $150 \mathrm{mM} \mathrm{NaCl}, 1 \mathrm{mM}$ EDTA, $1 \%$ Triton X-100, proteinase inhibitor cocktail (Roche Applied Sciences) and halt phosphatase inhibitor cocktail (Thermo Scientific), and subjected to immunoprecipitation with a monoclonal anti-BCL2 antibody (Santa Cruz Biotechnology, 1:50). Eluates were separated by SDS-PAGE and detected by anti-beclin 1 antibody (Santa Cruz Biotechnology, 1:200) and anti-BCL2-HRP antibody (C2, Santa Cruz Biotechnology, 1:100) and autoradiography.

\section{Mouse exercise studies}

For acute exercise studies, 8-week-old (wild type, BCL2 AAA and $\operatorname{Atg} 1611^{\mathrm{HM}}$ ) or 12-weekold $\left(B e c n I^{+/-}\right.$and wild-type $B e \mathrm{Cn}^{+/+}$littermate) mice were acclimated to and trained on a $10^{\circ}$ uphill Exer 3/6 open treadmill (Columbus Instruments) for 2 days. On day 1 mice ran for $5 \mathrm{~min}$ at $8 \mathrm{~m} \mathrm{~min}^{-1}$ and on day 2 mice ran for $5 \mathrm{~min}$ at $8 \mathrm{~m} \mathrm{~min}^{-1}$ followed by another 5 $\mathrm{min}$ at $10 \mathrm{~m} \mathrm{~min}^{-1}$. On day 3 , mice were subjected to a single bout of running starting at the speed of $10 \mathrm{~m} \mathrm{~min}^{-1}$. Forty minutes later, the treadmill speed was increased at a rate of $1 \mathrm{~m}$ $\mathrm{min}^{-1}$ every $10 \mathrm{~min}$ for a total of $30 \mathrm{~min}$, and then increased at the rate of $1 \mathrm{~m} \mathrm{~min}^{-1}$ every 5 min until mice were exhausted. Exhaustion was defined as the point at which mice spent more than $5 \mathrm{~s}$ on the electric shocker without attempting to resume running. Total running time was recorded and total running distance was calculated for each mouse.

For long-term exercise training, male $B c 12^{\mathrm{WT}}$ or $B c l 2^{\mathrm{AAA}}$ mice ( 8 weeks old) were randomly divided into three cohorts, including: (1) mice fed a regular diet (Harlan Teklad) without daily exercise, (2) mice fed a HFD containing 60\% fat (Research Diets) without daily exercise, and (3) mice fed a HFD with daily exercise. Prior to initiation of exercise, mice were fed a HFD for 4 weeks. At the end of the fourth week, mice in the exercise groups were acclimated to treadmill running for 2 days as in the acute exercise studies, and then were trained on the treadmill with $10^{\circ}$ uphill incline for $50 \mathrm{~min} \mathrm{~d}^{-1}, 5 \mathrm{~d}_{\text {week }}{ }^{-1}$ at $17 \mathrm{~m}$ $\min ^{-1}$ for 8 weeks. Mice were given a HFD during the 8 -week training period. All animal procedures were performed in accordance with institutional guidelines and with approval from the Institutional Animal Care and Use Committee. 


\section{Autophagy analyses}

MEFs expressing GFP-LC3 were cultured in normal or starvation (EBSS, Earle's balanced salt solution) medium for $4 \mathrm{~h}$, and GFP-LC3 puncta were quantified by fluorescence microscopy as described ${ }^{27}$. For assessment of autophagy in vivo following starvation or exercise, 8-week-old $B c 12^{\mathrm{WT}}$ or $B c 12^{\mathrm{AAA}}$ GFP-LC3 mice or $B e c n 1^{+/+}$or $\mathrm{Becn}^{+/-}$GFPLC3 mice were either subjected to starvation for $48 \mathrm{~h}$ or exercised for the indicated time period, anaesthetized by isoflurane, and perfused with $4 \%$ paraformaldehyde (PFA). Skeletal muscle (vastus lateralis, tibialis anterior, extensor digitorum longus and soleus), heart (left ventricle), pancreas and liver tissues were fixed in 4\% PFA overnight, $15 \%$ sucrose for $4 \mathrm{~h}$ and 30\% sucrose overnight before frozen sections were prepared. The number of GFP-LC3 puncta per unit area of tissue was quantified by fluorescence microscopy as described ${ }^{15}$. Autophagy in skeletal and cardiac muscle, liver, pancreas and adipose tissue during baseline conditions and after exercise was also analysed by western blot analysis of tissue extracts with antibodies against LC3 and p62 (see below for details).

\section{Immunofluorescence studies}

For immunofluorescence staining of frozen muscle and pancreatic sections, slides were heated at $50{ }^{\circ} \mathrm{C}$ for $10 \mathrm{~min}$, rehydrated in $100 \%, 95 \%$ and $70 \%$ ethanol, washed $3 \times 5 \mathrm{~min}$ in PBS, blocked with $1 \%$ goat serum and immunostained with a rabbit anti-mouse GLUT4 antibody (Alpha Diagnostic, 1:100 dilution) (muscle samples) and an Alexa Fluor 594 donkey anti-rabbit secondary antibody (Invitrogen, 1:1,000 dilution) or with a guinea pig anti-swine insulin antibody (Dako, 1:500 dilution) (pancreatic samples) and an Alexa Fluor 594 goat anti-guinea pig secondary antibody (Invitrogen, 1: 500 dilution).

Immunofluorescence studies on skeletal muscle sections with anti-laminin and anti-type I myosin heavy chain antibodies were performed as previously described ${ }^{28}$.

Immunofluorescence images were taken using a Zeiss Axioplan2 microscope or Leica TCS SP5 confocal microscope.

\section{Muscle morphology and function studies}

Sections were stained with haematoxylin and eosin to visualize tissue architecture following standard protocols. Metachromatic ATPase staining and measurement of fibre crosssectional area were performed as previously described ${ }^{28}$. Periodic acid-Schiff staining for carbohydrates was performed according to standard protocols ${ }^{29}$. Succinic dehydrogenase staining on cryosections was carried out using a $0.2 \mathrm{M}$ phosphate buffer at $\mathrm{pH}$ 7.6. For staining, $270 \mathrm{mg}$ succinic acid and $10 \mathrm{mg}$ nitro blue tetrazolium were freshly dissolved in 10 $\mathrm{ml}$ phosphate buffer and incubated with sections for $1 \mathrm{~h}$.

To measure muscle strength, a modified mesh grip assay was applied ${ }^{30}$. Briefly, mice were placed on the wired mesh of cage tops. When they firmly grabbed the mesh, the cage top was flipped and held at a height of $30 \mathrm{~cm}$ above the bench until the mice released their grip. The time for each mouse to remain on the mesh was recorded. TUNEL staining of muscle sections to detect apoptosis was performed according to the manufacturer's instructions (ApopTag Peroxidase In situ Apoptosis Detection Kit, Millipore), using Sigma FAST3,3' diaminobenzidine (DAB) tablets as the peroxidase substrate.

\section{Electron microscopy}

Mice were euthanized, and liver and pancreas were rapidly fixed in $2.5 \%$ glutaraldehyde in $0.1 \mathrm{M}$ cacodylate buffer. Electron microscopy was performed as previously described ${ }^{31}$. 


\section{Mouse metabolic studies and blood tests}

Food intake, $\mathrm{O}_{2}$ consumption, $\mathrm{CO}_{2}$ production, heat production and physical activity were measured in TSE metabolic chambers (TSE Systems) over a 4 day period. Mice in the exercise group were removed from the chambers and allowed to run for 50 min daily as in the above protocol during the day (light period). Metabolic measurements were performed for $12 \mathrm{~h}$ daily during the dark period. Fat mass and lean tissue mass were determined using the Bruker Minispec mq10 scan system. Plasma glucose, lactate, cholesterol and triglycerides were measured using the Vitros 250 system (Ortho-Clinical Diagnostics). Plasma insulin (Crystal Chem), serum leptin (Crystal Chem) and adiponectin (Millipore) levels were determined using commercial ELISA kits. Glucose tolerance tests were performed by oral glucose injection $\left(1 \mathrm{~g} \mathrm{~kg}^{-1}\right)$ after a $3 \mathrm{~h}$ fast. Blood was drawn from tail veins at the indicated time points after glucose injection and serum glucose levels were analysed using commercial glucose assay reagents (Sigma, Cat\# 6918, P7119, F5803).

\section{Echocardiography}

Cardiac function was assessed by echocardiography, using a Visualsonic Vevo 2100 ultrasound machine equipped with a 30-Mhz transducer applied to the chest wall. Ventricular dimensions and ejection fraction were assessed using short axis view in twodimensional and three-dimensional modes using the onboard VisualSonics cardiac analysis package.

\section{Muscle glucose uptake assays}

Surgical catheterization of the jugular vein was performed on each mouse. At day 4 after catheterization, mice were transferred to clean cages at 9:00 a.m. to begin a $4 \mathrm{~h}$ fast. Mice then ran on a treadmill for $50 \mathrm{~min}$ using the exercise protocol described above. At $t=50$ min, a $13 \mu \mathrm{Ci}$ bolus of ${ }^{14} \mathrm{C}$-deoxyglucose was injected intravenously and mice resumed running for another $25 \mathrm{~min}$ according to the exercise protocol described above before euthanasia. Skeletal muscle (soleus) and brain tissues were dissected and homogenized in $1.5 \mathrm{ml} 0.5 \%$ perchloric acid. ${ }^{14} \mathrm{C}$-deoxyglucose counts were determined in homogenized samples as previously described ${ }^{32}$ and muscle values were normalized to the counts in the brain.

\section{Western blot analyses}

Mouse tissue extracts were prepared by homogenizing tissues in lysis buffer containing 50 $\mathrm{mM}$ Tris (pH 7.9), $150 \mathrm{mM} \mathrm{NaCl}, 1 \mathrm{mM}$ EDTA, $1 \%$ Triton X-100, proteinase inhibitor cocktail (Roche Applied Sciences) and halt phosphatase inhibitor cocktail (Pierce), and subjected to western blot analysis with anti-LC3 (Novus Biologicals, 1:500), anti-p62 (BD Biosciences, 1:500 dilution; Progen (C-terminal specific), 1:1,000 dilution). Anti-p-CaMKI (Santa Cruz Biotechnology, 1:100), anti-CaMKI (Santa Cruz Biotechnology, 1:300), antiBCL2 (Santa Cruz Biotechnology, 1: 100), anti-AMPK (Cell Signaling, 1:1,000), anti-pAMPK (Cell Signaling, 1:1,000), anti-JNK (Cell Signaling, 1:400), anti-p-JNK (Cell Signaling, 1:500) anti-AS160 (Millipore, 1:400) anti-p-AS160 (Millipore, 1:100), anti-4EBP1 (Cell Signaling, 1:1,000), anti-p-4E-BP1 (Cell Signaling, 1:1,000), anti-p-ACC (Millipore, 1:500) anti-AKT (Cell Signaling, 1:600), anti-p-AKT (Cell Signaling, 1:500), anti-p38 (Cell Signaling, 1:500), anti-p-p38 (Cell Signaling, 1:400), anti-atrogin-1 (ECM Biosciences, 1:400), ATG16L1 (Sigma, 1:1,000) and anti-actin (Santa Cruz Biotechnology, $1: 3,000)$ antibodies.

For in vitro assessment of AMPK activation, MEFS derived from $B c 12^{\mathrm{AAA}}$ and $B c l 2^{\mathrm{WT}}$ mice, $B e c n 1^{+/-}$and $B e n c 1^{+/+}$mice, and $A \operatorname{tg} 1611^{\mathrm{HM}}$ and $A \operatorname{tg} 1611^{\mathrm{WT}}$ mice were treated with $2 \mathrm{mM}$ of the AMP analogue AICAR, or DMSO vehicle for $2 \mathrm{~h}$. Whole-cell lysates were 
prepared, and AMPK and phospho-AMPK levels were determined by western blot analysis. For quantification, the relative intensity values of western blot bands were normalized to that of the first lane (set as 1) in the wild-type non-exercised group.

\section{Statistical analyses}

ANOVA approaches were used to compare values among different experimental groups for data that met the normality assumption. One-way ANOVA was used for comparison between two groups. Two-way ANOVA was used for comparison of the magnitude of changes between two different groups in mice of two different genotypes. The normality assumption for the ANOVA model was checked using residual plots. When the assumption was violated, the data were log-transformed in order to meet the assumption. For data sets in which $\log$ transformation was inadequate to meet the analysis assumption, the nonparametric Wilcoxon rank-sum test was used.

\section{Real-time PCR analyses}

RNA isolation from muscle tissues and real-time RT-PCR were performed as previously described $^{28}$. The following Sybr Green primers were used: $\beta$-actin: forward, CTGGCTCCTAGCACCATGAAGAT; reverse, GGTGGACAGTGAGGCCAGGAT; UCP1: forward, TCAGGATTGGCCTCTA CGAC; reverse, TAAGCCGGCTGAGATCTTGT; UCP2: forward, TGCCCGTA ATGCCATTGTC; reverse, AGTGGCAAGGGAGGTCATCT; cytochrome c: forward, GTCTGTTCGGGCGGAAGACAG; reverse, GGGGAGAGGATAC CCTGATGG; and cytochrome b: forward, ATTCATTGACCTACCTGCCC; reverse, TCTGATGTGTAGTGTATGGC. The following Tagman primers were used: PPARGC1a (PGC1a): Mm00447183_m1; myoglobin: Mm00442968_m1; PPARGC1 $\beta$ (PGC1 $\beta$ ): Mm01258518_m1; MYH7 (MHC Type I): Mm00600555_ml; MYH1 (MHC Type IId/x): Mm01332488_g1; MYH2 (MHC Type IIa): Mm01332564_m1; and GAPDH: Mm99999915_g1.

\section{Supplementary Material}

Refer to Web version on PubMed Central for supplementary material.

\section{Acknowledgments}

We thank the UT Southwestern Mouse Metabolic Phenotyping Core and E. Berglund for assistance with metabolic measurements, J. Shelton for assistance with muscle stains, N. Mizushima for critical reagents, and B. D. Levine for expert advice. This work was supported by National Institutes of Health grants RO1 CA109618 (B.L.), ROI HL080244 (J.A.H.), ROI HL090842 (J.A.H.), ROI AI084887 (H.W.V.), RCI DK086629 (P.E.S.), RO1 CA112023 (P.E.S.) and 1PO1 DK0887761 (P.E.S.).

\section{References}

1. Handschin C, Spiegelman BM. The role of exercise and PGC1a in inflammation and chronic disease. Nature. 2008; 454:463-469. [PubMed: 18650917]

2. Mizushima N, Levine B. Autophagy in mammalian development and differentiation. Nature Cell Biol. 2010; 12:823-830. [PubMed: 20811354]

3. Kuma A, Mizushima N. Physiological role of autophagy as an intracellular recycling system: with an emphasis on nutrient metabolism. Semin. Cell Dev. Biol. 2010; 21:683-690. [PubMed: 20223289]

4. Levine B, Kroemer G. Autophagy in the pathogenesis of disease. Cell. 2008; 132:27-42. [PubMed: 18191218] 
5. Yang L, Li P, Fu S, Calay ES, Hotamisligil GS. Defective hepatic autophagy in obesity promotes ERstress and causes insulinresistance. Cell Metab. 2010; 11:467-478. [PubMed: 20519119]

6. Ebato C, et al. Autophagy is important in islet homeostasis and compensatory increase of $\beta$ cell mass in response to high-fat diet. Cell Metab. 2008; 8:325-332. [PubMed: 18840363]

7. Mizushima N, Yamamoto A, Matsui M, Yoshimori T, Ohsumi Y. In vivo analysis of autophagy inresponse tonutrient starvation using transgenic mice expressing a fluorescent autophagosome marker. Mol. Biol. Cell. 2004; 15:1101-1111. [PubMed: 14699058]

8. He C, Levine B. The Beclin 1 interactome. Curr. Opin. Cell Biol. 2010; 22:140-149. [PubMed: 20097051]

9. Wei Y, Pattingre S, Sinha S, Bassik M, Levine B. JNK1-mediated phosphorylation of Bcl-2 regulates starvation-induced autophagy. Mol. Cell. 2008; 30:678-688. [PubMed: 18570871]

10. Goodyear LJ, Kahn BB. Exercise, glucose transport, and insulin sensitivity. Annu. Rev. Med. 1998; 49:235-261. [PubMed: 9509261]

11. Ryder JW, Chibalin AV, Zierath JR. Intracellular mechanisms underlying increases in glucose uptake in response to insulin or exercise in skeletal muscle. Acta Physiol. Scand. 2001; 171:249257. [PubMed: 11412137]

12. Zisman A, et al. Targeted disruption of the glucose transporter 4 selectively in muscle causes insulin resistance and glucose intolerance. Nature Med. 2000; 6:924-928. [PubMed: 10932232]

13. Hardie DG. AMP-activated protein kinase: an energy sensor that regulates all aspects of cell function. Genes Dev. 2011; 25:1895-1908. [PubMed: 21937710]

14. Bassik MC, Scorrano L, Oakes SA, Pozzan T, Korsmeyer SJ. Phosphorylation of BCL-2 regulates $\mathrm{ER} \mathrm{Ca}^{2+}$ homeostasis and apoptosis. EMBO J. 2004; 23:1207-1216. [PubMed: 15010700]

15. Qu X, et al. Promotion of tumorigenesis by heterozygous disruption of the beclin 1 autophagy gene. J. Clin. Invest. 2003; 112:1809-1820. [PubMed: 14638851]

16. Malik SA, et al. BH3 mimetics activate multiple pro-autophagic pathways. Oncogene. 2011; 30:3918-3928. [PubMed: 21460857]

17. Cadwell K, et al. A key role for autophagy and the autophagy gene Atg1611 in mouse and human intestinal Paneth cells. Nature. 2008; 456:259-263. [PubMed: 18849966]

18. Fujita N, et al. The Atg16L complex specifies the site of LC3 lipidation for membrane biogenesis in autophagy. Mol. Biol. Cell. 2008; 19:2092-2100. [PubMed: 18321988]

19. Asterholm IW, Scherer PE. Enhanced metabolic flexibility associated with elevated adiponectin levels. Am. J. Pathol. 2010; 176:1364-1376. [PubMed: 20093494]

20. Zhang Y, et al. Positional cloning of the mouse obese gene and its human homologue. Nature. 1994; 372:425-432. [PubMed: 7984236]

21. Scherer PE, Williams S, Fogliano M, Baldini G, Lodish HF. A novel serum protein similar to C1q, produced exclusively in adipocytes. J. Biol. Chem. 1995; 270:26746-26749. [PubMed: 7592907]

22. Frederich RC, et al. Leptin levels reflect body lipid content in mice: evidence for diet-induced resistance to leptin action. Nature Med. 1995; 1:1311-1314. [PubMed: 7489415]

23. Van Heek M, et al. Diet-induced obese mice develop peripheral, but not central, resistance to leptin. J. Clin. Invest. 1997; 99:385-390. [PubMed: 9022070]

24. Shetty S, Kusminski CM, Scherer PE. Adiponectin in health and disease: evaluation of adiponectin-targeted drug development strategies. Trends Pharmacol. Sci. 2009; 30:234-239. [PubMed: 19359049]

25. Almind K, Manieri M, Sivitz WI, Cinti S, Kahn CR. Ectopic brown adipose tissue in muscle provides a mechanism for differences in risk of metabolic syndrome in mice. Proc. Natl Acad. Sci. USA. 2007; 104:2366-2371. [PubMed: 17283342]

26. Su T, et al. Deletion of histidinetriad nucleotide-binding protein 1/PKC-interacting protein in mice enhances cell growth and carcinogenesis. Proc. Natl Acad. Sci. USA. 2003; 100:7824-7829. [PubMed: 12810953]

27. Furuya N, Yu J, Byfield M, Pattingre S, Levine B. The evolutionarily conserved domain of Beclin 1 is required for Vps34 binding, autophagy and tumor suppressor function. Autophagy. 2005; 1:46-52. [PubMed: 16874027] 
28. Moresi V, et al. Myogenin and class II HDACs control neurogenic muscle atrophy by inducing E3 ubiquitin ligases. Cell. 2010; 143:35-45. [PubMed: 20887891]

29. Sheehan, DC.; Hrapchak, DC. Theory and Practice of Histotechnology. 2nd edn. Battelle; 1980. p. 162-166.

30. Butchbach M, Edwards J, Burghes A. Abnormal motor phenotype in the SMN $\Delta 7$ mouse model of spinal muscular atrophy. Neurobiol. Dis. 2007; 27:207-219. [PubMed: 17561409]

31. Liang XH, et al. Induction of autophagy and inhibition of tumorigenesis by beclin 1. Nature. 1999; 402:672-676. [PubMed: 10604474]

32. Berglund ED, et al. Fibroblast growth factor 21 controls glycemia via regulation of hepatic glucose flux and insulin sensitivity. Endocrinology. 2009; 150:4084-4093. [PubMed: 19470704] 

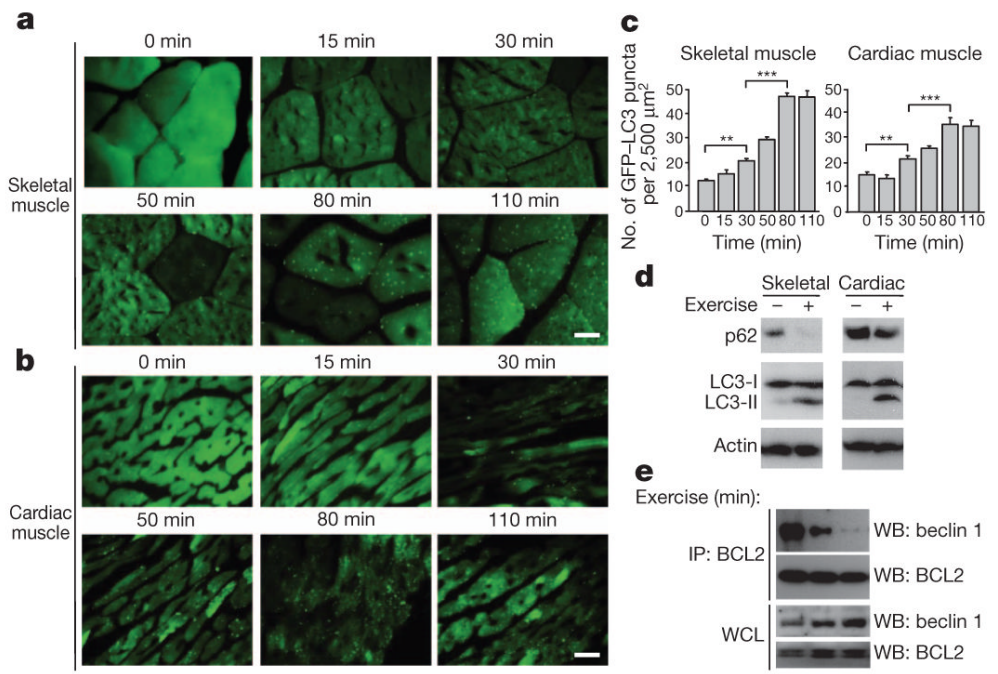

Figure 1. Exercise induces autophagy in skeletal and cardiac muscle

a, b, Representative images of GFP-LC3 puncta (autophagosomes) in skeletal (vastus lateralis) (a) and cardiac (b) muscle from GFP-LC3 transgenic mice at serial time points after exercise. Scale bar, $20 \mu \mathrm{m}$. c, Quantification of data (mean \pm s.e.m. of 10 tissue sections) in a and b. $* * P<0.01, * * * P<0.001$ (one-way ANOVA). d, Western blot analysis of LC3-I/II (non-lipidated and lipidated forms of MAP1LC3, respectively) and p62 levels in indicated tissue from mice at rest $(-)$ or after maximal exercise $(+)$. Skeletal and cardiac indicate skeletal and cardiac muscle, respectively. e, Co-immunoprecipitation of beclin 1 with BCL2 in muscle tissue from mice at indicated time points after exercise. IP, immunoprecipitate; WB, western blot; WCL, whole cell lysates. 


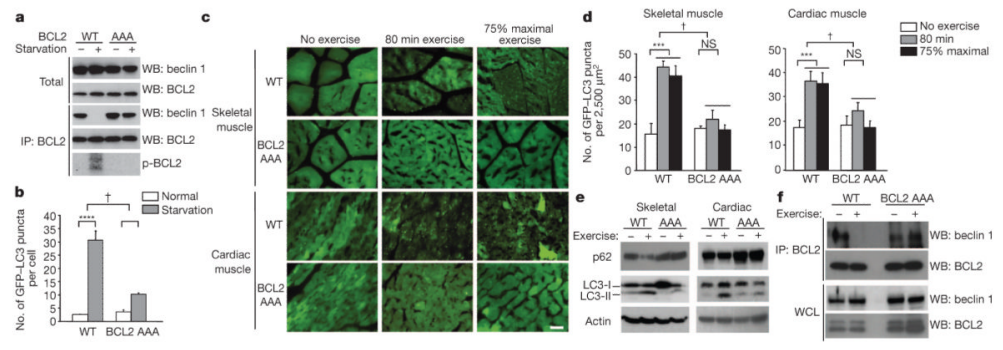

Figure 2. Non-phosphorylatable BCL2 AAA knock-in mutations block BCL2 phosphorylation, BCL2-beclin 1 dissociation, and starvation- and exercise-induced autophagy

a, Analysis of BCL2 phosphorylation (detected by anti-BCL2 immunoprecipitation and autoradiography of ${ }^{32} \mathrm{P}$-labelled cells) and beclin 1 co-immunoprecipitation with BCL2 in wild-type (WT) or BCL2 AAA MEFs grown in normal media or subjected to $4 \mathrm{~h}$ Earle's balanced salt solution (EBSS) starvation. p-BCL2, phospho-BCL2. b, Quantification of GFP-LC3 puncta (autophagosomes) in MEFs of indicated genotype in normal growth conditions or starvation conditions. Data represent mean \pm s.e.m. for 100 cells per well of triplicate samples per condition. Similar results were observed in three independent experiments. c, Representative images of GFP-LC3 puncta (autophagosomes) in skeletal and cardiac muscle of GFP-LC3 wild-type and GFP-LC3 BCL2 AAA mice before exercise, after 80 min exercise, or after $75 \%$ of maximal exercise capacity. Scale bar, $20 \mu \mathrm{m}$. d, Quantification of data (mean \pm s.d. of 4 mice per experimental group) in c. e, Western blot analysis of LC3-I/II and p62 levels in indicated tissue from mice of indicated genotype at rest (-) or after maximal exercise (+). Skeletal and cardiac indicate skeletal and cardiac muscle, respectively. f, Co-immunoprecipitation of beclin 1 with BCL2 in muscle tissue from mice of indicated genotype at rest (-) or after $30 \mathrm{~min}$ of exercise. WCL, whole cell lysates. NS, not significant. $* P<0.05$, $* * P<0.01$, $* * * P<0.001$, one-way ANOVA for comparison between groups; $† P<0.001$, two-way ANOVA for comparison of magnitude of changes between different groups in mice of different genotypes. 

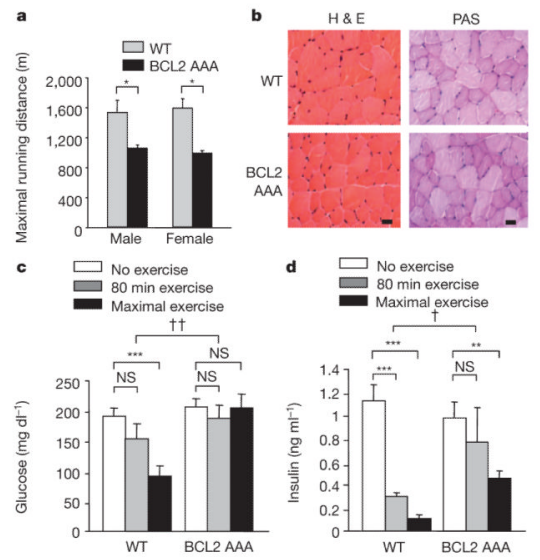

d $\square$ No exercise
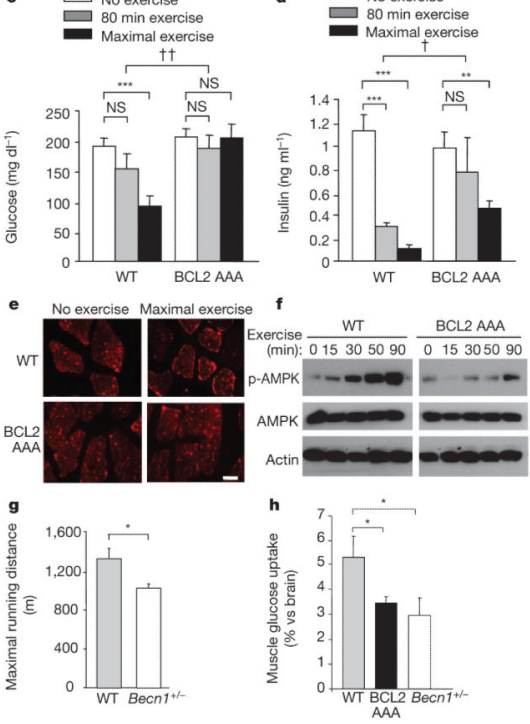

Figure 3. BCL2 AAA mice show deficient exercise endurance and alterations in muscle glucose metabolism

a, Maximal treadmill running distance for mice of indicated genotype. Data represent mean \pm s.e.m. of 5 mice per group. b, Representative haematoxylin and eosin (H \& E) and periodic acid-Schiff (PAS) staining in tibialis anterior muscle sections from mice of indicated genotype. Scale bar, $20 \mu \mathrm{m}$. c, d, Plasma glucose (c) and insulin (d) levels in mice of indicated genotype at rest, after 80 min exercise $(\sim 900 \mathrm{~m})$, or maximal exercise. Data represent combined mean \pm s.e.m. for 9-11 mice per group from three independent cohorts; similar results were observed in each cohort. e, Representative images of GLUT4 immunofluorescence staining in vastus lateralis muscle of mice of indicated genotype before and after maximal exercise. Scale bar, $20 \mu \mathrm{m}$. For $\mathbf{b}$ and $\mathbf{e}$, similar results were observed in 3 mice per group. f, Western blot analysis of AMPK phosphorylation (p-AMPK (Thr 172)) in vastus lateralis muscle lysates from mice of indicated genotype at indicated time after exercise. g, Maximal treadmill running distance for mice of indicated genotype. Data represent mean \pm s.e.m. of 4-6 male mice per group. $\mathbf{h}$, Soleus muscle ${ }^{14} \mathrm{C}$-deoxyglucose uptake during treadmill exercise in mice of indicated genotype. Data represent mean \pm s.e.m. of 3 mice per group. $* P<0.05, * * P<0.01$, $* * * P<0.001$, one-way ANOVA for comparison between groups; $\uparrow P<0.05$, $\dagger \dagger P<0.01$, two-way ANOVA for comparison of magnitude of changes between different groups in mice of different genotypes. NS, not significant. 

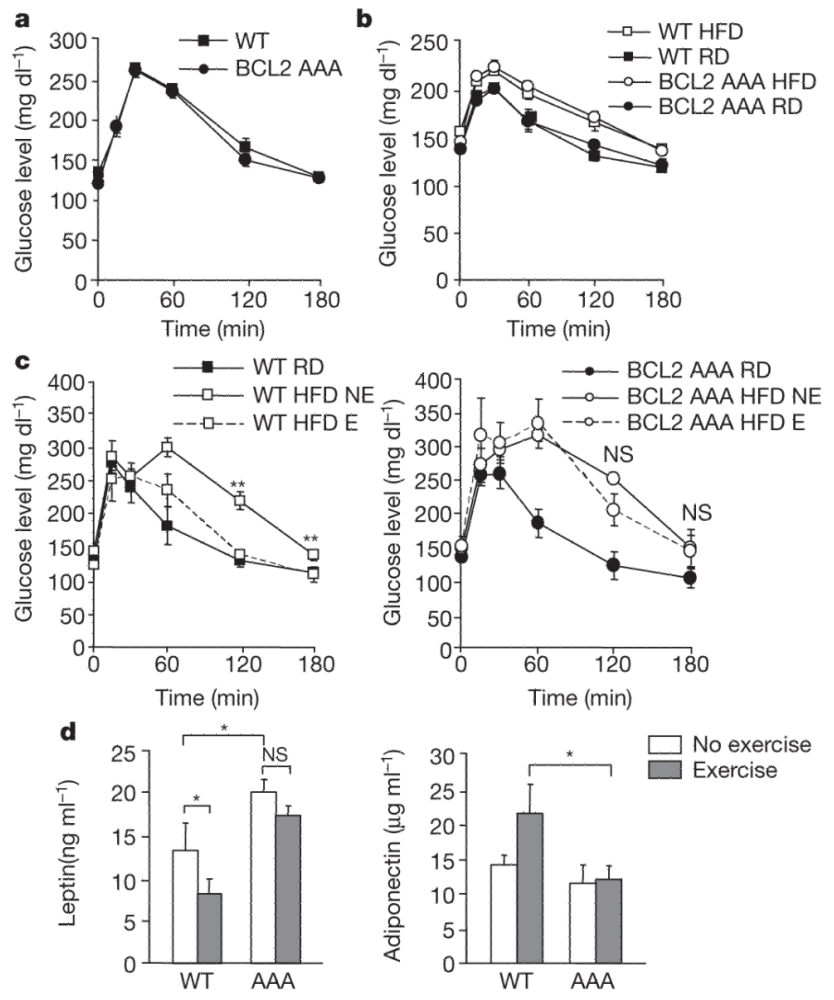

Figure 4. Long-term exercise training protects wild-type but not BCL2 AAA mice from HFDinduced glucose intolerance

a, b, Oral glucose tolerance test (OGTT) before (a, week 0) and after (b, week 4) 4 weeks of HFD. c, d, OGTT (c) and serum leptin and adiponectin levels (d) after 8 weeks of daily exercise. For a-d, results represent the mean \pm s.e.m. for 4-5 mice per group. E, exercise; NE, no exercise; RD, regular diet. $* P<0.05$, $* * P<0.01$, (c, one-way ANOVA; d, Wilcoxon rank test). NS, not significant. 